\title{
Optimal Least-Squares FIR Digital Filters for Compensation of Chromatic Dispersion in Digital Coherent Optical Receivers
}

\author{
Amir Eghbali, Håkan Johansson, Oscar Gustafsson and Seb J. Savory
}

\section{Linköping University Post Print}

\section{Tweet}

N.B.: When citing this work, cite the original article.

Original Publication:

Amir Eghbali, Håkan Johansson, Oscar Gustafsson and Seb J. Savory, Optimal Least-Squares FIR Digital Filters for Compensation of Chromatic Dispersion in Digital Coherent Optical Receivers, 2014, Journal of Lightwave Technology, (32), 8, 1449-1456.

http://dx.doi.org/10.1109/JLT.2014.2307916

Copyright: Optical Society of America http://www.osa.org/

Postprint available at: Linköping University Electronic Press http://urn.kb.se/resolve?urn=urn:nbn:se:liu:diva-106013 


\title{
Optimal Least-Squares FIR Digital Filters for Compensation of Chromatic Dispersion in Digital Coherent Optical Receivers
}

\author{
Amir Eghbali, Member, IEEE, Håkan Johansson, Senior Member, IEEE, Oscar Gustafsson, Senior Member, IEEE, \\ and Seb J. Savory, Senior Member, IEEE
}

\begin{abstract}
This paper proposes optimal finite-length impulse response (FIR) digital filters, in the least-squares (LS) sense, for compensation of chromatic dispersion (CD) in digital coherent optical receivers. The proposed filters are based on the convex minimization of the energy of the complex error between the frequency responses of the actual CD compensation filter and the ideal CD compensation filter. The paper utilizes the fact that pulse shaping filters limit the effective bandwidth of the signal. Then, the filter design for CD compensation needs to be performed over a smaller frequency range, as compared to the whole frequency band in the existing CD compensation methods. By means of design examples, we show that our proposed optimal LS FIR CD compensation filters outperform the existing filters in terms of performance, implementation complexity, and delay.
\end{abstract}

Index Terms - Chromatic dispersion (CD), digital filter, fiber optics, optimal least-squares (LS) FIR filter.

\section{INTRODUCTION}

I $\mathrm{N}$ optical fibers, the group velocity of the propagating signal is frequency dependent and optical pulses hence spread in time. This results in CD [1]-[5] thus limiting the transmission distance and/or data rate [6]. The $\mathrm{CD}$ is traditionally compensated using optical devices with opposite dispersion [5] but such approaches cannot be easily tuned/improved to accommodate different fiber spans/properties and quality measures [6].

With coherent detection schemes, richer constellations, and fast analog to digital converters (ADCs), digital signal processing is playing a growing role in CD compensation [4], [6], [7]. In digital coherent optical receivers, $\mathrm{CD}$ is modeled as a frequency response given by [1]-[5]

$$
C\left(e^{j \omega T}\right)=e^{-j K(\omega T)^{2}}, \quad K=\frac{D \lambda^{2} z}{4 \pi c T^{2}} .
$$

Here, $D, \lambda, z$, and $c$ are the fiber dispersion parameter, wavelength, propagation distance, and the speed of light, respectively. This paper uses $\omega T=2 \pi f T$ to represent the "digital frequency" with a sampling period of $T$ thus corresponding to a

Manuscript received October 9, 2013; revised January 19, 2014 and February 18, 2014; accepted February 19, 2014. Date of publication February 23, 2014; date of current version March 9, 2014.

A. Eghbali, H. Johansson, and O. Gustafsson are with the Division of Electronics Systems, Department of Electrical Engineering, Linköping University, 58183 Linköping, Sweden (e-mail: amire@isy.liu.se; hakanj@isy.liu.se; oscarg@isy.liu.se).

S. J. Savory is with the Optical Networks Group, Department of Electronic and Electrical Engineering, University College London, London, WC1E 7JE, U.K. (e-mail: s.savory@ee.ucl.ac.uk).

Digital Object Identifier 10.1109/JLT.2014.2307916 sampling frequency of $F=\frac{1}{T}$. The $\mathrm{CD}$ can be compensated by designing a filter $H\left(e^{j \omega T}\right)$ to approximate a desired frequency response as

$$
H_{D e s}\left(e^{j \omega T}\right)=\frac{1}{C\left(e^{j \omega T}\right)}=e^{j K(\omega T)^{2}} .
$$

This is also referred to as static channel equalization [2] for which FIR or infinite-length impulse response (IIR) filters ${ }^{1}$ can be used [1], [2], [4]. This paper uses FIR filters because they (i) are unconditionally stable [8], (ii) can efficiently be implemented in the frequency domain [2], and (iii) do not have limitations on the maximal sampling frequency, as opposed to their IIR counterparts [9].

\section{A. Contribution of the Paper and Relation to Previous Work}

In [1] and [2], an FIR CD compensation filter was derived with a complex impulse response given by

$$
h(n)=\sqrt{\frac{j}{4 K \pi}} e^{-j \frac{n^{2}}{4 K}}, \quad-\left\lfloor\frac{N}{2}\right\rfloor \leq n \leq\left\lfloor\frac{N}{2}\right\rfloor
$$

where the length of the filter $h(n)$ is odd and given ${ }^{2}$ as [1]

$$
N=2\lfloor 2 K \pi\rfloor+1 .
$$

To decrease the hardware cost, it is generally of interest to reduce $N$ which in turn reduces the number of arithmetic operations required for implementation [8]. Besides hardware cost, a smaller $N$ decreases the overall delay of the filter which is an important issue, especially, in high-speed communications.

A drawback of (3) is that it does not utilize the effects of pulse shaping filters in limiting the bandwidth of signals. With band-limited signals, at the output of pulse shaping filters, we do not need to compensate $\mathrm{CD}$ over the whole frequency band of $\omega T \in[-\pi, \pi]$. In other words, and as we will see in Section III-B2, the band-limiting properties of pulse shaping filters allow us to compensate $\mathrm{CD}$ in a smaller frequency band thereby reducing the filter length and the implementation cost. As discussed later in Section III-A2, (3) has another drawback because it does not necessarily improve the $\mathrm{CD}$ compensation quality if we increase the filter length. As we will also describe in Sections III-A1 and III-B2, a third drawback of (3) is that it has suboptimal performance, especially for modulation formats with higher spectral-efficiencies.

\footnotetext{
${ }^{1}$ The FIR filter is also called feed-forward equalizer or transversal tap filter [2].

${ }^{2}$ Note that (4) does not guarantee a desired performance, e.g., bit error rate (BER), for a given $K$.
} 
With the ever increasing demands on optical communication systems, to move towards high-speed traffic using highspectral-efficiency modulation formats, previous CD compensation approaches need to be revisited so as to (i) improve the CD compensation quality, (ii) relax the demands on the subsequent adaptive equalizers, and (iii) overcome the above-mentioned drawbacks.

This paper proposes optimal FIR filters, in the LS sense, which outperform (3). Our proposed filters can be designed to compensate $\mathrm{CD}$ in either a small frequency band or the whole frequency band. By increasing the filter length, our proposed filters can obtain arbitrarily good CD compensation, even with modulation formats having high spectral-efficiencies. As will be seen in Section III-B2, considering the effects of pulse shaping filters, in our proposed method, we can further reduce the implementation cost. Even without considering the effects of pulse shaping filters, i.e., for the whole frequency band and the same filter length, our proposed filters still outperform (3), as we will see in Sections III-A1 and III-A2.

In Section IV-A, we will also compare our proposed filters to those obtained by the frequency sampling method (FSM). We will show that our proposed filters require fewer number of taps, than those of FSM, in order to obtain the same BER performance. Further, we will show that the superiority, of our proposed filters, becomes more pronounced at modulation formats with a high spectral-efficiency.

\section{B. Paper Outline}

Section II introduces the proposed optimal LS FIR filters for $\mathrm{CD}$ compensation whereas the numerical results are discussed in Section III. Some design and implementation issues are discussed in Section IV. Finally, the concluding remarks are given in Section V.

\section{Proposed CD COMPENSATION FILTERS}

Consider a complex FIR filter of length $N_{c}$, which like $N$ is assumed to be odd here, with a frequency response as [8]

$$
H\left(e^{j \omega T}\right)=\sum_{n=-\frac{N_{c}-1}{2}}^{\frac{N_{c}-1}{2}} h(n) e^{-j n \omega T} .
$$

To measure the accuracy of the designed filter $H\left(e^{j \omega T}\right)$, which approximates $H_{D e s}\left(e^{j \omega T}\right)$ in (2), we define the energy of the complex error as, for $\omega T \in\left[\Omega_{1}, \Omega_{2}\right]$,

$$
E=\frac{1}{2 \pi} \int_{\Omega_{1}}^{\Omega_{2}}\left|H\left(e^{j \omega T}\right)-H_{D e s}\left(e^{j \omega T}\right)\right|^{2} d(\omega T) .
$$

To obtain $h(n)$, we formulate an LS problem to find an FIR filter $\hat{\mathbf{h}}$ which minimizes $E$ as [10]

$$
\hat{\mathbf{h}}=\operatorname{argmin} E
$$

where $\hat{\mathbf{h}}$ is defined by (8) as shown at the bottom of the page. With signals having a flat frequency spectrum, (7) amounts to minimizing the error vector magnitude. The solution of (7) is unique and globally optimal in the LS sense. ${ }^{3}$ It is given by

$$
\hat{\mathbf{h}}=\mathbf{Q}^{-1} \mathbf{D}
$$

where $\mathbf{Q}$ is an $N_{c} \times N_{c}$ matrix with elements as

$$
\begin{aligned}
Q(n, m) & =\frac{1}{2 \pi} \int_{\Omega_{1}}^{\Omega_{2}} e^{j(n-m) \omega T} d(\omega T) \\
& =\frac{e^{-j(-n+m) \Omega_{1}}-e^{-j(-n+m) \Omega_{2}}}{2 j \pi(-n+m)} \\
& = \begin{cases}\frac{\Omega_{2}-\Omega_{1}}{2 \pi} & n=m \\
\frac{e^{-j(-n+m) \Omega_{1}}-e^{-j(-n+m) \Omega_{2}}}{2 j \pi(-n+m)} & n \neq m\end{cases}
\end{aligned}
$$

for $0 \leq n \leq N_{c}-1$ and $0 \leq m \leq N_{c}-1$. Note that $\mathbf{Q}$ is a Hermitian Toeplitz matrix and it thus suffices to only compute its first row with $n=0$ in (10). This property of $\mathbf{Q}$ reduces the design complexity by reducing the number of computations required in (10). Further, $\mathbf{D}$ is defined as in (11) as shown at the bottom of the page, with

$$
\begin{aligned}
D(n) & =\frac{1}{2 \pi} \int_{\Omega_{1}}^{\Omega_{2}} H_{D e s}\left(e^{j \omega T}\right) e^{j n \omega T} d(\omega T) \\
& =\frac{1}{2 \pi} \int_{\Omega_{1}}^{\Omega_{2}} e^{j \omega T(K \omega T+n)} d(\omega T) .
\end{aligned}
$$

After some manipulations, (12) gives the closed form solution of (13) as shown at the bottom of the page, in which $\operatorname{erf}(\alpha)$ is

\footnotetext{
${ }^{3}$ By optimality, we mean that for a given filter length $N_{c}$, no other filter (having the same length) will result in an $E$ which is smaller than that obtained by $\hat{\mathbf{h}}$ in (9).
}

$$
\hat{\mathbf{h}}=\left[\begin{array}{lllll}
\hat{h}\left(-\frac{N_{c}-1}{2}\right) & \hat{h}\left(-\frac{N_{c}-1}{2}+1\right) & \ldots & \hat{h}\left(\frac{N_{c}-1}{2}-1\right) & \hat{h}\left(\frac{N_{c}-1}{2}\right)
\end{array}\right]^{\mathrm{T}}
$$

$$
\mathbf{D}=\left[\begin{array}{lllll}
D\left(-\frac{N_{c}-1}{2}\right) & D\left(-\frac{N_{c}-1}{2}+1\right) & \ldots & D\left(\frac{N_{c}-1}{2}-1\right) & D\left(\frac{N_{c}-1}{2}\right)
\end{array}\right]^{\mathbf{T}}
$$

$$
D(n)=\frac{e^{-j\left(\frac{n^{2}}{4 K}+\frac{3 \pi}{4}\right)}}{4 \sqrt{\pi K}}\left(\operatorname{erf}\left(\frac{e^{j \frac{3 \pi}{4}}(2 K \pi-n)}{2 \sqrt{K}}\right)+\operatorname{erf}\left(\frac{e^{j \frac{3 \pi}{4}}(2 K \pi+n)}{2 \sqrt{K}}\right)\right),-\frac{N_{c}-1}{2} \leq n \leq \frac{N_{c}-1}{2}
$$




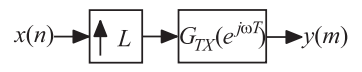

(a)

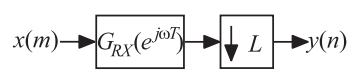

(b)

Fig. 1. Interpolation and decimation by L. (a) Interpolation. (b) Decimation.

defined as

$$
\operatorname{erf}(\alpha)=\frac{2}{\sqrt{\pi}} \int_{0}^{\alpha} e^{-t^{2}} d t
$$

Note that if $\alpha$, in (14), is an imaginary term, we obtain the imaginary error function erfi $(\alpha)$ which is related to $\operatorname{erf}(\alpha)$ as

$$
\operatorname{erfi}(\alpha)=\frac{\operatorname{erf}(j \alpha)}{j}=\frac{1}{j} \frac{2}{\sqrt{\pi}} \int_{0}^{j \alpha} e^{-t^{2}} d t
$$

There exist efficient numerical methods to evaluate (15), e.g., [11]. As discussed earlier, (3) targets the whole frequency band where $\Omega_{2}=-\Omega_{1}=\pi$. Utilizing the properties of pulse shaping filters, the values of $\Omega_{2}$ and $\Omega_{1}$ (and hence $N_{c}$ ) can be reduced as will be discussed in Section II-A, below.

\section{A. Effects of Pulse Shaping}

Pulse shaping comprises interpolation (decimation) at the transmitter (receiver) side and it reduces the inter-carrier interference and inter-symbol interference. In Fig. 1(a) [1(b)], interpolation [decimation] by the integer factor $L>1$ requires an upsampler [a downsampler] and an anti-imaging [anti-aliasing] filter $G_{T X}\left(e^{j \omega T}\right)\left[G_{R X}\left(e^{j \omega T}\right)\right]$ [12]. These filters usually have lowpass characteristics ${ }^{4}$ with a roll-off of $0<\rho<1$ leading to a stopband edge at $\omega_{s} T=\pi \frac{1+\rho}{L}$. They are designed so that $\sum_{l=0}^{L-1} G_{T X}\left(e^{j\left(\omega T-\frac{2 \pi l}{L}\right)}\right) G_{R X}\left(e^{j\left(\omega T-\frac{2 \pi l}{L}\right)}\right) \approx 1$ [13]. A typical solution is the square-root raised cosine filter as [14]

$$
g_{T X}(t)=g_{R X}(t)=\frac{\sin \left(\frac{\pi t}{T}(1-\rho)\right)+\frac{4 \rho t}{T} \cos \left(\frac{\pi t}{T}(1+\rho)\right)}{\frac{\pi t}{T}\left(1-\left(\frac{4 \rho t}{T}\right)^{2}\right)} .
$$

This paper deals with digital filters and we will hence use $t=\frac{n T}{L}$ in (16). As the interpolated signals have limited bandwidths, one does not actually need to compensate $\mathrm{CD}$ in the whole frequency band of $\omega T \in[-\pi, \pi]$. It suffices to design $\hat{\mathbf{h}}$ for the frequency band of $\omega T \in\left[-\omega_{s} T, \omega_{s} T\right]$ by using $\Omega_{2}=-\Omega_{1}=\omega_{s} T$ in (6)(12).

With $\Omega_{2}=-\Omega_{1}=\omega_{s} T$, one should however add a proper nonzero term to $\mathbf{Q}$ so as to avoid ill-conditioned matrices. One way to do so is to rewrite (9) as

$$
\hat{\mathbf{h}}=\left(\mathbf{Q}+\epsilon \mathbf{I}_{N_{c}}\right)^{-1} \mathbf{D}
$$

\footnotetext{
${ }^{4}$ Pulse shaping filters generally belong to a class of filters called $L$ th-band filters for which the transition band includes $\frac{\pi}{L}$ and the passband/stopband edges are equally distanced from $\frac{\pi}{L}$. Therefore, having passband/stopband edges as $\pi \frac{1 \mp \rho}{L}$ is a customary way of defining these edges so that they, with $0<\rho<1$ and integer $L>1$, are guaranteed to be smaller than $\pi$ which is necessary for digital filters [8], [12].
}

TABLE I

SIMULATION PARAMETERS USED IN EXAMPLES 1 AND 2

\begin{tabular}{|c|c|c|c|c|}
\hline Ex. & $N$ & $z \mathrm{~km}$ & $F \mathrm{~Hz}$ & $K$ \\
\hline 1 & 251 & 4000 & $21.4 \times 10^{9}$ & 19.9227 \\
\hline 2 & 875 & 1000 & $80 \times 10^{9}$ & 69.605 \\
\hline
\end{tabular}

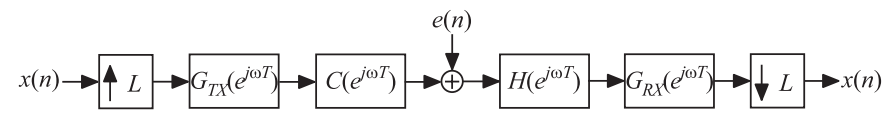

Fig. 2. Simulation chain composed of pulse shaping, CD model, and CD compensation filter.

where $\mathbf{I}_{N_{c}}$ is an $N_{c} \times N_{c}$ identity matrix which corresponds to the (approximately) unit energy of the CD compensation filter. By using $\epsilon$, we add a penalty term which is proportional to the unit energy of the CD compensation filter. Without this penalty term, the frequency response of the $\mathrm{CD}$ compensation filter can have undesired behaviors in the frequency range of $\omega T \in\left[\omega_{s} T, \pi\right]$. For each $\epsilon$, (17) gives an optimal filter but the best solution is dependent on $\epsilon$ as we will see in Section III-B1.

\section{NUMERICAL RESULTS}

We here assume the same parameters ${ }^{5}$ given in Table I. Note that Example 1 uses the same parameters as in [1]. However, for Example 2, we have increased the symbol rate $F$, while using the same oversampling ratio $L$, to show that our proposed filters can actually be used for future high-speed communications requiring longer $\mathrm{CD}$ compensation filters. In Example 1, (4) gives $N=251$ whereas for Example 2, we get $N=875$. Based on these parameters, we will compare the existing CD compensation filter, defined by (3), with our proposed filters in (9) or (17). This comparison is carried out using a simulation chain, shown in Fig. 2, where $e(n)$ represents the additive white Gaussian noise (AWGN) channel. In Section IV-A, we will also compare our proposed filters with those obtained by FSM.

We can add other noise sources to this simulation setup, e.g., laser phase noise [16] or impairments of ADCs and digital to analog converters (DACs). However, the CD compensation filters are independent of such effects [1] and we have therefore not considered them ${ }^{6}$. In (16), we use $\rho=0.25$ and we have chosen high orders for $G_{T X}\left(e^{j \omega T}\right)$ and $G_{R X}\left(e^{j \omega T}\right)$ so that the errors, arising from pulse shaping, are negligible. Then, the only error sources are those due to (i) the CD model, (ii) the CD compensation filter, and (iii) the AWGN channel.

Our Monte Carlo simulations use quadrature amplitude modulation (QAM) symbols. In the BER plots, the term "BTB" stands for the back-to-back propagation obtained with $C\left(e^{j \omega T}\right)=1$ and $H\left(e^{j \omega T}\right)=1$. In other words, BTB stands

\footnotetext{
${ }^{5}$ We can further reduce the implementation complexity by choosing $1<L<$ 2 but this would require interpolation/decimation by rational factors [15] and is beyond the scope of this paper. The main focus of this paper is to compare the performance of different filter design methods and as long as the simulation chain is the same, the choice of $L$ is not crucial.

${ }^{6}$ This paper compares different digital filters which compensate the same amount of $\mathrm{CD}$ and we assume other parts of the system to have a negligible effect. If we add other impairments, like laser phase noise or ADC/DAC errors, the system performance may ultimately be limited by other factors.
} 


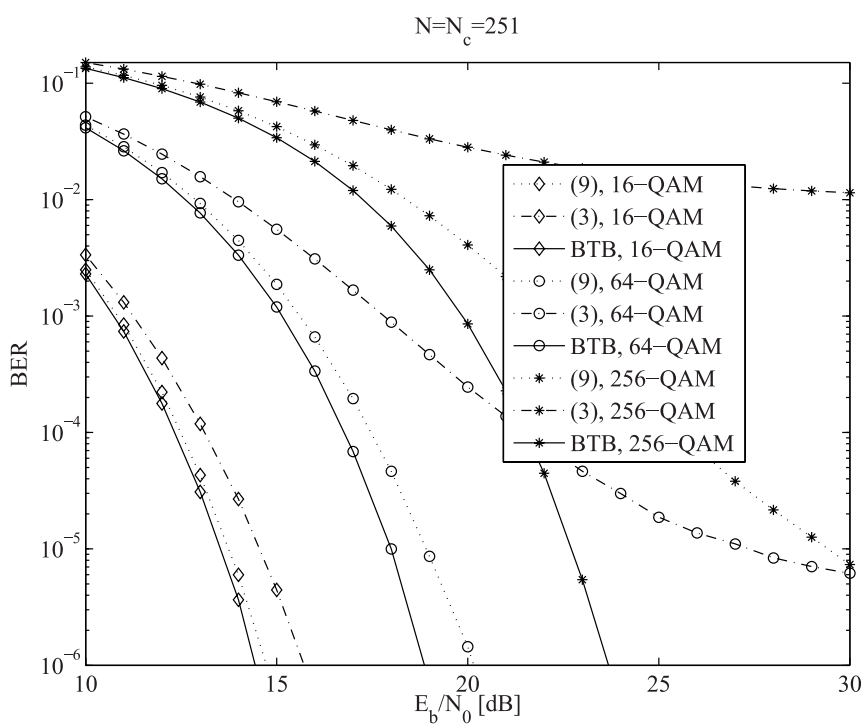

Fig. 3. Simulated uncoded BER for QAM data with $K=19.9227, L=$ $2, \Omega_{2}=-\Omega_{1}=\pi$, and the filters in (9) and (3).

for the matched filter performance without the CD effects. In our Monte Carlo simulations, we model $C\left(e^{j \omega T}\right)$ by solving a problem like (7) with $H_{D e s}\left(e^{j \omega T}\right)=C\left(e^{j \omega T}\right)$ but (as for the pulse shaping filters) we have chosen a high order so that the corresponding error becomes negligible.

\section{A. Fullband Case}

In this section, we will compare the filters in (9) and (3) using $\Omega_{2}=-\Omega_{1}=\pi$ and $L=2$.

1) BER Performance With the Same Filter Lengths: In this case, (10) becomes

$$
\begin{aligned}
Q(n, m) & = \begin{cases}1 & n=m \\
\frac{\sin ((n-m) \pi)}{(n-m) \pi} & n \neq m\end{cases} \\
& = \begin{cases}1 & n=m \\
0 & n \neq m .\end{cases}
\end{aligned}
$$

The matrix $\mathbf{Q}$ is thus an identity matrix and (9) hence amounts to only finding $\mathbf{D}$ in (11) and (12). This simplifies the design complexity, associated with (9), as it gives

$$
\hat{h}(n)=D(n)
$$

which is given by (13). Figs. 3 and 4 respectively compare the simulated uncoded BER of Examples 1 and 2 over an AWGN channel. As noted earlier, we use the parameters in Table I along with (4) to estimate $N$. With the same number ${ }^{7}$ of taps per $1000 \mathrm{ps} / \mathrm{nm}$ of $\mathrm{CD}$, our proposed filter, in (9), gives a smaller BER as compared to that of (3).

Figs. 3 and 4 show that beyond certain values of $\frac{E_{b}}{N_{0}}$ and for modulations with a high spectral-efficiency, the BER curves,

\footnotetext{
${ }^{7}$ In Example 1 and with $N=N_{c}=251$, we need $\frac{N}{z}=3.7$ taps per $1000 \mathrm{ps} / \mathrm{nm}$ of CD [1].
}

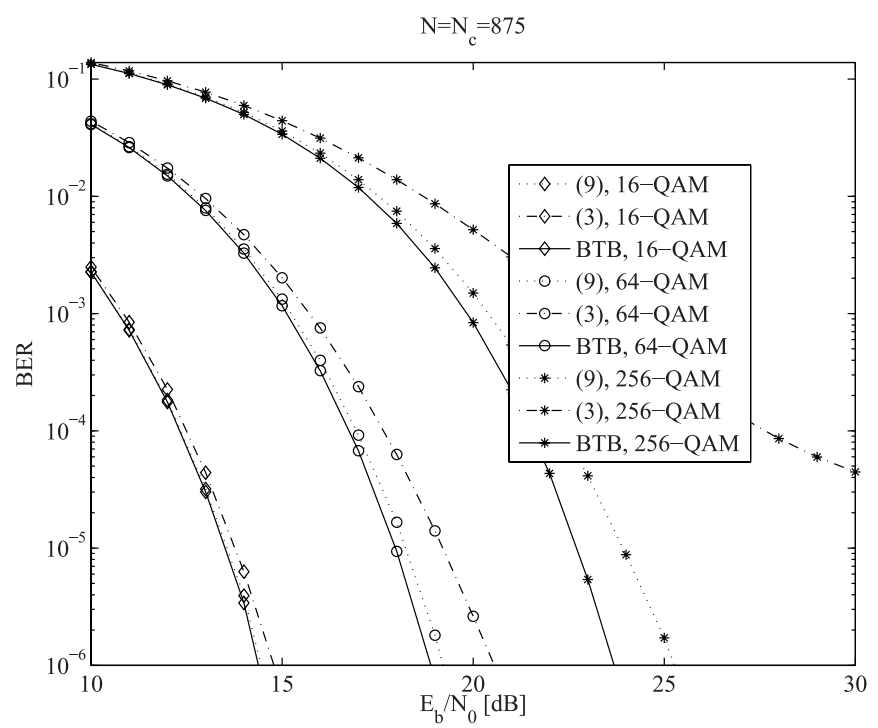

Fig. 4. Simulated uncoded BER for QAM data with $K=69.605, L=$ $2, \Omega_{2}=-\Omega_{1}=\pi$, and the filters in (9) and (3).

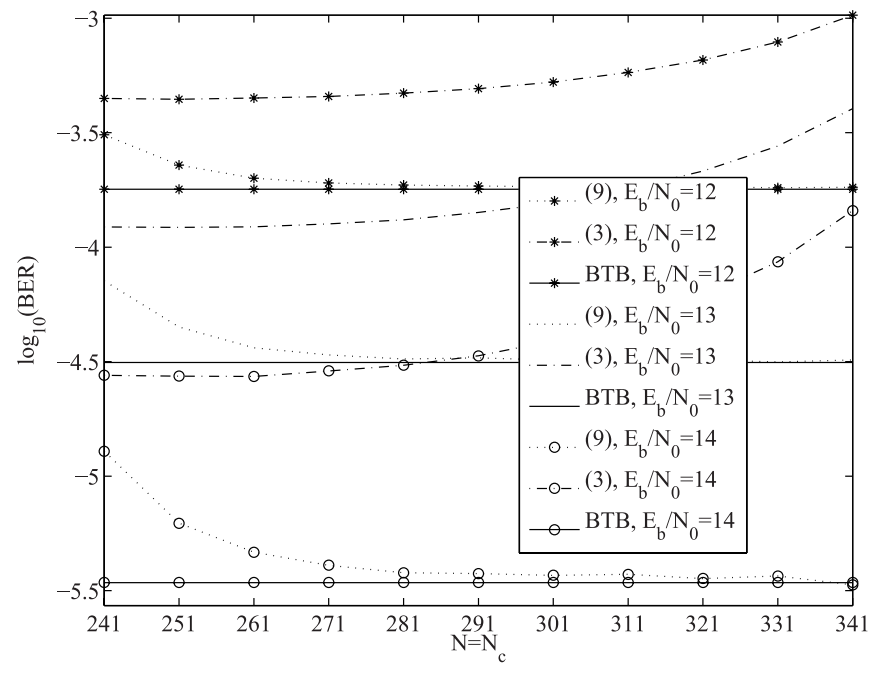

Fig. 5. Simulated uncoded BER values of 16-QAM data with different values of $N=N_{c}$ in (3) and (9) for $\Omega_{2}=-\Omega_{1}=\pi, L=2, K=19.9227$, and some values of $\frac{E_{b}}{N_{0}} \mathrm{~dB}$.

for (3), tend to obtain a floor ${ }^{8}$. These plots also show that for simpler modulation schemes and with large BER values, the filters obtained by (9) and (3) result in roughly similar BER curves. For much simpler modulation schemes, e.g., 4-QAM, the BER curves of (9) and (3), are very similar and we have thus not plotted them. However, with modulation formats having higher spectral-efficiencies and if a small BER is desired, the filter obtained by (9) clearly has a better performance.

2) Comparison With Different Filter Lengths: As noted earlier, a drawback of (3) is that CD compensation does not necessarily improve if we increase the filter length. Fig. 5 shows the uncoded BER values of 16-QAM data where we have considered different values of $N=N_{c}$ in (3) and (9) as well as

\footnotetext{
${ }^{8}$ The BER curves of (9) will also become flat if $\frac{E_{b}}{N_{0}}$ further increases. However, with (3), the BER curves become flat at much lower values of $\frac{E_{b}}{N_{0}}$.
} 
(a)

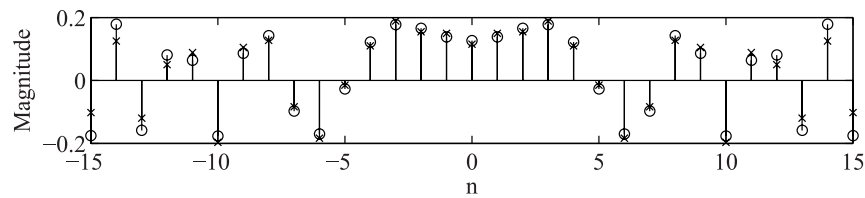

(b)

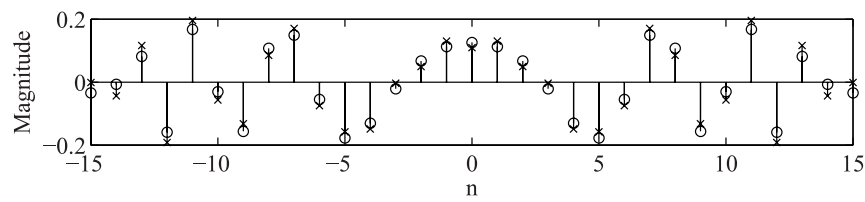

(c)

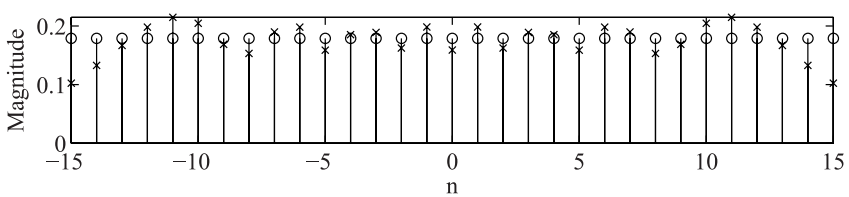

Fig. 6. The real parts, the imaginary parts, and the absolute values of the impulse responses $h(n)$ and $\hat{h}(n)$ with $N=N_{c}=31$ and $z=500 \mathrm{~km}$ along with the parameters of Example 1. The plot with circles represents $h(n)$. (a) Real part. (b) Imaginary part. (c) Absolute value.

different values of $\frac{E_{b}}{N_{0}}$. As can be seen, if we increase $N_{c}$ in (9), the value of BER decreases. This means that we can obtain arbitrarily good CD compensation filters by increasing $N_{c}$. However, this does not apply to (3) and the value of BER may even increase if we increase $N$.

In conclusion, the filters given by (9) not only can be designed to obtain arbitrarily good CD compensation but they also require fewer taps for the same BER performance. The reason is two-fold with the first being that (9) gives a smaller $E$, than (3), for the same filter length. This is a direct result (see Footnote 3 ) of optimality of (9). The second reason is that (this is easy to verify using, e.g., MATLAB) the value of $E$ decreases if the length of the filter, given by (9), is increased. However, this does not necessarily apply to the filter in (3). Note that if $E$ becomes very small, i.e., smaller than the noise of the AWGN channel, the BER curve does not improve even if we increase $N_{c}$. The BER curves, of (9), hence asymptotically reach the BER curve of BTB propagation. According to Fig. 5, such a phenomenon does not happen for the BER curves of (3) and beyond certain values of $N$, the BER even degrades.

3) Relationships Between Closed Form Impulse Responses: A comparison of (3) and (9) [with its closed form solution in (19)] reveals that the impulse response values are partly similar. For example, the term $e^{-j \frac{n^{2}}{4 K}}$ appears in both (3) and (19). To compare the values of $h(n)$ and $\hat{h}(n)$, Fig. 6 shows the real parts, the imaginary parts, and the absolute values of these impulse responses. For illustration purposes, we have considered a smaller value of $N=N_{c}=31$, obtained from $z=500 \mathrm{~km}$ and the parameters of Example 1. Note that the absolute value of $h(n)$, in (3), has a constant value as $|h(n)|=\frac{1}{2 \sqrt{K \pi}}$. However, this does not apply to $\hat{h}(n)$. Also, Fig. 7 shows the magnitude responses and the group delays of the impulse responses in Fig. 6. As can be seen, $\hat{h}(n)$ results in fewer overshoots in the magnitude re- (a)

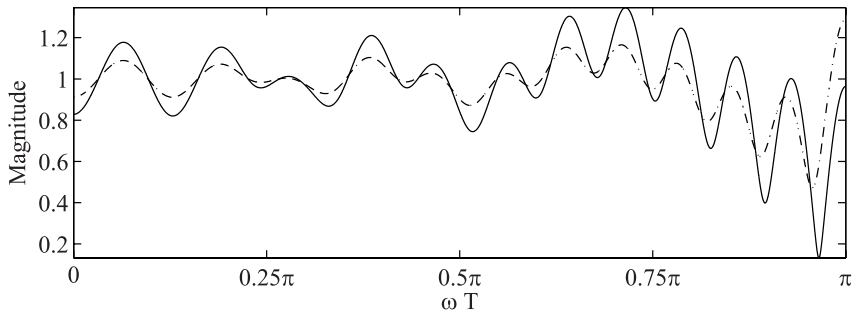

(b)

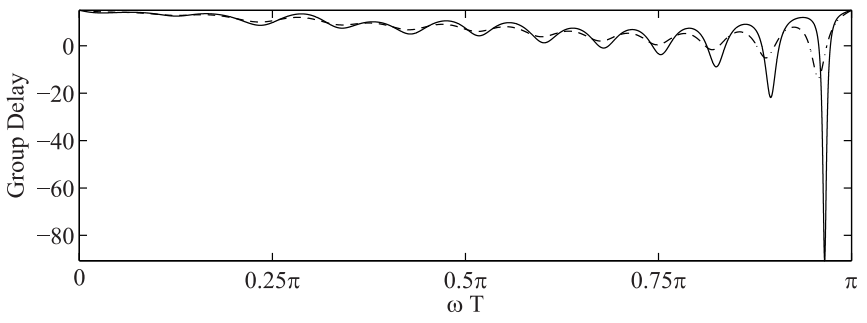

Fig. 7. Magnitude response and group delay of the filters obtained by (9) and (3) with $N=N_{c}=31$ and $z=500 \mathrm{~km}$ along with the parameters of Example 1. The solid line represents the filter obtained by (3).

(a)

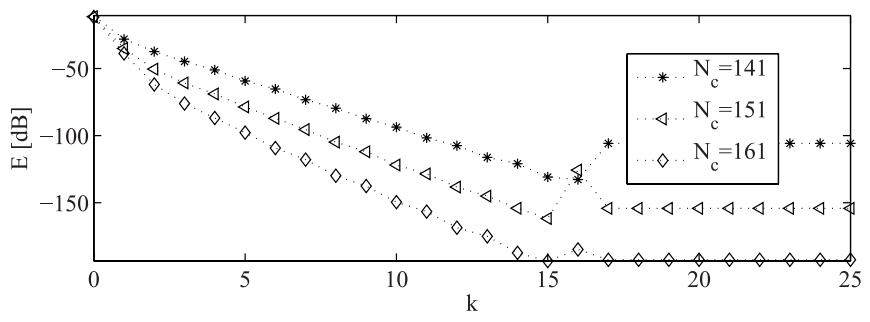

(b)

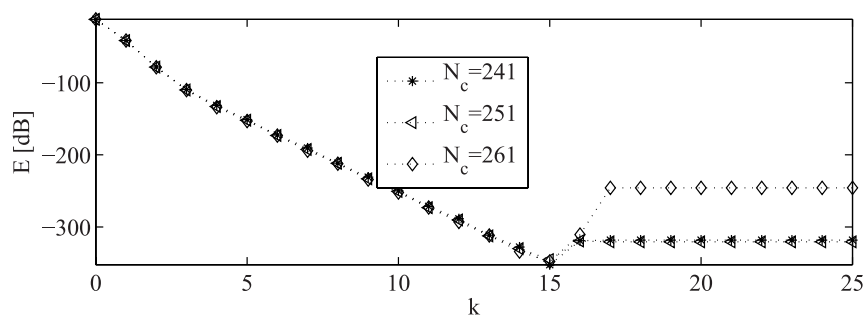

Fig. 8. The values of $E$, in (6), obtained from (17), $L=2, \Omega_{2}=-\Omega_{1}=$ $\frac{\pi(1+\rho)}{L}, K=19.9227$, and $\epsilon=10^{-k}$.

sponse and the group delay. Therefore, the group delay of $\hat{h}(n)$ tends to be more constant as opposed to that of $h(n)$. This is another explanation for the superiority of (9) over (3).

\section{B. Band-Limited Case}

In this section, we will compare the filters in (17) and (3) using $\Omega_{2}=-\Omega_{1}=\frac{\pi(1+\rho)}{L}$ and $L=2$.

1) Choice of Penalty Factor: As discussed earlier, the penalty factor $\epsilon$, in (17), should have a small nonzero value but very small values of $\epsilon$ may result in numerical problems and must be avoided. Based on our experiments, in MATLAB, Fig. 8 depicts the values of $E$, in (6), where we have used (17) along 


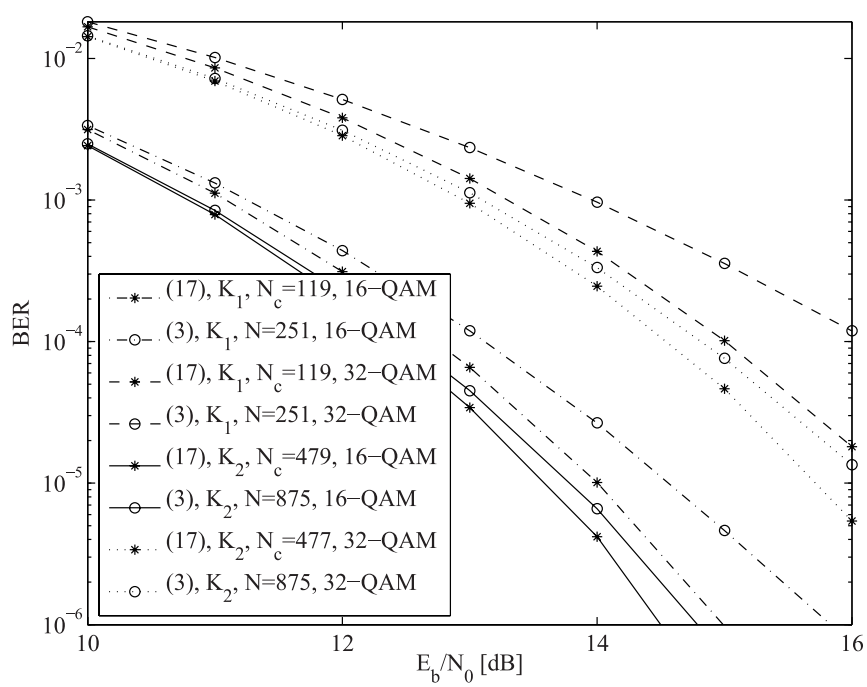

Fig. 9. Simulated uncoded BER for QAM data in Example 1 and 2 along with $\Omega_{2}=-\Omega_{1}=\frac{\pi(1+\rho)}{L}, L=2$, and $\epsilon=10^{-14}$. Here, $K_{1}$ and $K_{2}$ stand for the values of $K$ in Example 1 and 2, respectively.

with $L=2, K=19.9227$, and $\epsilon=10^{-k}, k=0,1, \ldots, 25$ for some values of $N_{c}$. Our numerical results show that if $\epsilon<10^{-15}$, the value of $E$ may be degraded as numerical problems occur due to the software precision limits. For any set $\left\{N_{c}, K, L\right\}$, one must however find the best value of $\{\epsilon, E\}$ so as to improve the CD compensation quality. For a given set $\left\{N_{c}, K, L\right\}$, the best value of $\{\epsilon, E\}$ can be determined by a simple exhaustive search which amounts to designing $\hat{h}(n)$ for different values of $\epsilon$ and selecting the one which gives the lowest $E$. Note that we, here, only illustrate the trend of $E$ for some choices of $\epsilon$ and $N_{c}$. To obtain a desired performance, say a given BER, and if $K$ is large, we would require a longer filter. Our simulations in Fig. 8, however, aim to show how to choose $\epsilon$ and how the software (MATLAB in this case) precision affects this choice.

For very small values of $E$, the performance of the system, e.g., BER, is anyhow mainly determined by other factors like the AWGN channel. Therefore, minor changes in $\epsilon$ and $N_{c}$ will not be crucial although small values of $N_{c}$ and $E$ are always desired. Our experiments show that below certain ${ }^{9}$ small values of $\epsilon$, say when moving from $\epsilon=10^{-12}$ to $\epsilon=10^{-14}$, the lowest possible $N_{c}$ may vary by a small value, e.g., two. In our simulations, we have therefore used $\epsilon=10^{-14}$ and we have ignored such minor changes in $N_{c}$.

2) BER Performance With Different Filter Lengths: In this case, $\mathbf{Q}$ is a general Hermitian Toeplitz matrix. Fig. 9 compares the simulated uncoded BER of Examples 1 and 2 over an AWGN channel where we have assumed $\epsilon=10^{-14}$. In these figures, we report the lowest possible length, i.e., $N_{c}$ in (17), whose BER is smaller than or equal to the BER of $N$ in (3). For example, in Fig. 9 and with a 16-QAM data, we can select $N_{c}=119$ in (17) and still obtain a smaller BER as compared to the case with $N=251$ in (3). In case of Example 1 and with 16-QAM

\footnotetext{
${ }^{9}$ In general, for high-spectral-efficiency modulation formats with small BER values, the values of $\epsilon$ and $E$ must be smaller.
}

data, this shows a reduction of about $1-\frac{N_{c}}{N}=52$ percent in the number of taps per $1000 \mathrm{ps} / \mathrm{nm}$ of CD.

\section{DESIGN AND IMPLEMENTATION COMPLEXITY}

In digital filters, one generally has to consider two issues: design complexity and implementation complexity. The design complexity refers to the required (numerical) effort to obtain the filter coefficients. Here, we have two cases for comparison of the design complexity. If $\Omega_{2}=-\Omega_{1}=\pi$, (9) leads to a closed form solution as in (19). Therefore, for the same filter length and with $\Omega_{2}=-\Omega_{1}=\pi$, the design complexities of (3) and (9) are comparable.

For the case with $\Omega_{2}=-\Omega_{1}=\frac{\pi(1+\rho)}{L}$, (17) requires to compute $\mathbf{D}, \mathbf{Q}$, and $\left(\mathbf{Q}+\epsilon \mathbf{I}_{N_{c}}\right)^{-1}$. For the same filter length along with $\Omega_{2}=-\Omega_{1}=\frac{\pi(1+\rho)}{L}$, our proposed method thus has a higher design complexity than (3). However, as can clearly be seen from Fig. 9, the choice of $\Omega_{2}=-\Omega_{1}=\frac{\pi(1+\rho)}{L}$ allows to reduce $N_{c}$ which in turn reduces the overall design complexity. Our numerical results (not reported here) also show that if $L$ increases, the value of $N_{c}$ will be much smaller because, in such a case, the value of $\Omega_{2}=-\Omega_{1}=\frac{\pi(1+\rho)}{L}$ becomes smaller. Then, the design complexity of (17) will be even smaller. It is generally desired to derive accurate formulas for estimation of $N_{c}$ so as to guarantee a desired performance, e.g., BER. This requires many Monte Carlo simulations in a design space comprised of $\epsilon, \frac{E_{b}}{N_{0}}, K, L$, and the desired BER value and is beyond the scope of this paper. However, as can be seen from Fig. 9, $N_{c}$ can roughly be estimated as $\frac{N}{L}$. This corresponds to the band $\omega T \in\left[0, \omega_{s} T\right]$, over which the CD needs to actually be compensated.

Generally, CD compensation filters need not be designed very often. Thus, the major complexity-burden comes from their implementation rather than their design. By means of numerical examples, we have shown that our proposed filters, in (9) and (17), clearly require fewer number of taps [to obtain a given BER performance], than the filter in (3). Especially for modulation formats with severe requirements, the implementation complexity of our proposed filters will thus be lower than that of (3).

This paper deals with optimization-based filter design and we differentiate between filter design and filter implementation. In other words, we optimize the impulse response of the filter with constraints in the frequency domain. This is a common practice in the design of digital filters [8], [12]. For a given impulse response, one can generally implement the filter (or equivalently, the linear convolution) either in the time domain (e.g., see [8, Sec. VIII.3]) or in the frequency domain (e.g., see [8, Sec. V.10]). In the latter method, one can use, e.g., the overlap-add or overlap-save methods, to reduce the implementation complexity [8], [17], [18]. The implementation complexity can also be reduced in the time domain using, e.g., polyphase realizations or multiple constant multiplication methods [8], [12]. Here, we do not discuss such implementation issues as they (i) can be applicable to any given impulse response and (ii) do not affect the filtering performance. Instead, this paper has focused on the 


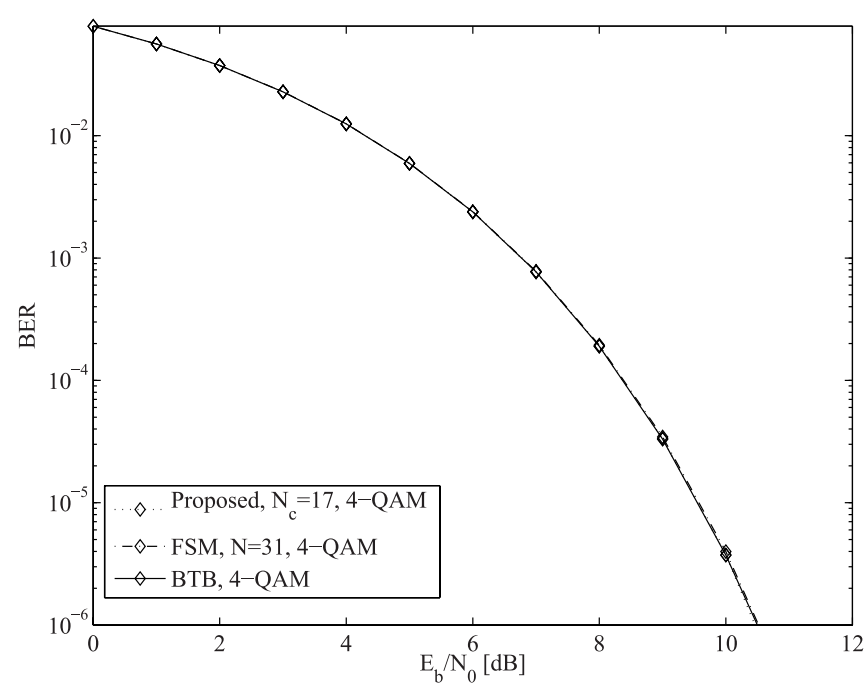

Fig. 10. Simulated uncoded BER for 4-QAM data in the example of Section III-A3 with $\Omega_{2}=-\Omega_{1}=\frac{\pi(1+\rho)}{L}, L=2$, and $\epsilon=10^{-14}$.

number of filter taps per $1000 \mathrm{ps} / \mathrm{nm}$ of CD. Regardless of how the filter is implemented, it is always beneficial to reduce the filter length from the design/implementation as well as the delay points of view.

\section{A. Comparison With Filters Designed Using FSM}

A straightforward alternative, to our proposed optimizationbased filter design method, is to use the inverse CD function at $N$ predefined uniformly-spaced frequencies $\omega_{k} T$ corresponding to those of a length- $N$ discrete Fourier transform (DFT), i.e., $\omega_{k} T=\frac{2 k \pi}{N}, k=0,1, \ldots, N-1$. Then, $\mathrm{CD}$ equalization is typically performed in the frequency domain with a filter frequency response represented as $H_{\mathrm{FSM}}(k)=e^{j K\left(\omega_{k} T\right)^{2}}, k=0,1, \ldots, N-1$. Still, there exists an underlying impulse response $h_{\mathrm{FSM}}(n)$ where $H_{\mathrm{FSM}}(k)=$ $\sum_{n=0}^{N-1} h_{\mathrm{FSM}}(n) e^{-j n \omega_{k} T}, k=0,1, \ldots, N-1$. This method corresponds to the so-called FSM which has a low design complexity. However, a drawback of FSM is that the CD is only (perfectly) equalized at $N$ predefined uniformly-spaced frequencies $\omega_{k} T$. In such methods, there is an exact control of the system behavior over these $N$ predefined uniformly-spaced frequencies $\omega_{k} T$. Between these frequencies, the behavior of the system cannot be controlled. This is in contrast to our proposed method which covers all frequencies through the integral in (6).

To illustrate this, let us revisit the example in Section III-A3. In this case, we can optimize our proposed filters, as in (17). For FSM, we can obtain the underlying impulse response $h_{\mathrm{FSM}}(n)$ through the inverse DFT of $H_{\mathrm{FSM}}(k)$. Then, we can compute $E$, in (6), in the frequency band of interest. With $N=N_{c}=31$, our proposed method gives $E=-220 \mathrm{~dB}$ whereas the filter obtained by FSM gives $E=-63 \mathrm{~dB}$. As can be seen, our proposed method gives a smaller $E$ which then allows $N_{c}$ to be reduced to obtain the same BER performance as that of FSM. Fig. 10 compares the simulated uncoded BER for filters obtained by our proposed method and FSM in the example of Section III-A3. As can be seen, with a simple modulation

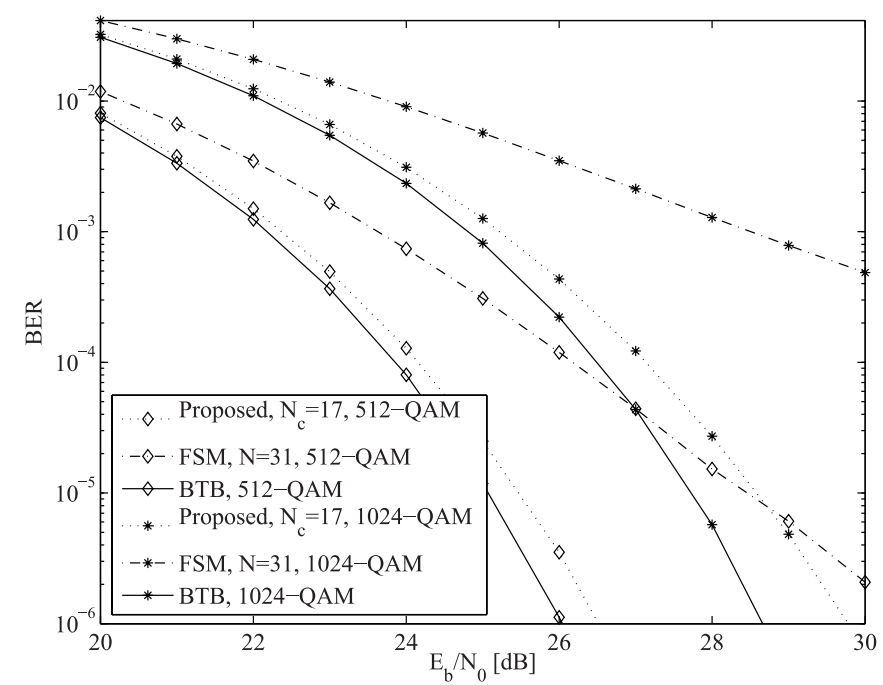

Fig. 11. Simulated uncoded BER for QAM data in the example of Section III-A3 with $\Omega_{2}=-\Omega_{1}=\frac{\pi(1+\rho)}{L}, L=2$, and $\epsilon=10^{-14}$.

format, like 4-QAM, both filters have a very good performance although our proposed method needs fewer number of taps to obtain the same BER as that of FSM. As discussed earlier, future optical communication systems will move towards high-speed traffic using high-spectral-efficiency modulation formats thus necessitating efficient CD compensation filters. Fig. 11 compares the simulated uncoded BER for filters obtained by our proposed method and FSM where we have considered some very high-spectral-efficiency modulation formats. As can be seen, our proposed method (with fewer number of taps) has clearly a better performance than FSM.

\section{CONCLUSION}

Optimal FIR digital filters, in the LS sense, for compensation of $\mathrm{CD}$ were derived. For the same amount of $\mathrm{CD}$, our proposed filters outperform (especially for modulation formats having high spectral-efficiencies) the exiting ones as they require fewer taps giving a lower implementation cost and delay. Design examples were provided for illustration and comparison. It was shown that considering the effects of pulse shaping filters, in our proposed method, we can further reduce the implementation cost.

\section{REFERENCES}

[1] S. J. Savory, "Digital filters for coherent optical receivers," Opt. Exp., vol. 16, no. 2, pp. 804-817, 2008.

[2] S. J. Savory, "Digital coherent optical receivers: Algorithms and subsystems," IEEE J. Sel. Topics Quantum Electron., vol. 16, no. 5, pp. 11641179, Sep./Oct. 2010.

[3] D. Lavery, R. Maher, D. S. Millar, B. C. Thomsen, P. Bayvel, and S. J. Savory, "Digital coherent receivers for long-reach optical access networks," J. Lightw. Technol., vol. 31, no. 4, pp. 609-620, Feb. 2013.

[4] G. Goldfarb and G. Li, "Chromatic dispersion compensation using digital IIR filtering with coherent detection," IEEE Photon. Technol. Lett., vol. 19, no. 13, pp. 969-971, Jul. 2007.

[5] G. P. Agrawal, Fiber-Optic Communication Systems. New York, NY, USA: Wiley, 2010.

[6] C. C. Davis and T. E. Murphy, "Fiber-optic communications," IEEE Signal Process. Mag., vol. 28, no. 4, pp. 152-150, Jul. 2011. 
[7] P. Bower and I. Dedic, "High speed converters and DSP for $100 \mathrm{G}$ and beyond," Opt. Fiber Technol., vol. 17, no. 5, pp. 464-471, May 2011.

[8] S. K. Mitra, Digital Signal Processing: A Computer Based Approach. New York, NY, USA: McGraw-Hill, Feb. 2006.

[9] M. Renfors and Y. Neuvo, "The maximum sampling rate of digital filters under hardware speed constraints," IEEE Trans. Circuits Syst., vol. 28, no. 3, pp. 196-202, Mar. 1980.

[10] S. S. Kidambi and R. P. Ramachandran, "Complex coefficient nonrecursive digital filter design using a least-squares method," IEEE Trans. Signal Process., vol. 44, no. 3, pp. 710-713, Mar. 1996.

[11] M. Abramowitz and I. A. Stegun, "Error function and Fresnel integrals," in Handbook of Mathematical Functions with Formulas, Graphs, and Mathematical Tables. New York, NY, USA: Dover, 1972, pp. 297-309.

[12] P. P. Vaidyanathan, Multirate Systems and Filter Banks. Englewood Cliffs, NJ, USA: Prentice-Hall, 1993.

[13] A. Eghbali, T. Saramäki, and H. Johansson, "On two-stage Nyquist pulse shaping filters," IEEE Trans. Signal Process., vol. 60, no. 1, pp. 483-488, Jan. 2012

[14] "Universal mobile telecommunications system (UMTS); base station (BS) radio transmission and reception (FDD)," 3GPP TS 25.104 version 6.8.0 Release 6, Dec. 2004

[15] A. Eghbali, H. Johansson, and P. Löwenborg, "A class of multimode transmultiplexers based on the Farrow structure," Circuits Syst. Signal Process., vol. 31, no. 3, pp. 961-985, Jun. 2012.

[16] W. Shieh and K.-P. Ho, "Equalization-enhanced phase noise for coherentdetection systems using electronic digital signal processing," Opt. Exp. vol. 16, no. 20, pp. $15718-15727,2008$.

[17] J. G. Proakis and D. G. Manolakis, Digital Signal Processing: Principles, Algorithms, and Applications, 4th ed. Upper Saddle River, NJ, USA: Prentice-Hall, 2007.

[18] V. K. Madisetti and D. B. Walliams, Eds., The Digital Signal Processing Handbook. Boca Raton, FL, USA: CRC Press, 1998.
Håkan Johansson (SM'06) received the Master of Science degree in computer science, and the Licentiate, Doctoral, and Docent degrees in electronics systems from Linköping University, Linköping, Sweden, in 1995, 1997, 1998, and 2001, respectively.

During 1998 and 1999, he held a Postdoctoral position at Signal Processing Laboratory, Tampere University of Technology, Tampere, Finland. He is currently a Professor of electronics systems in the Department of Electrical Engineering, Linköping University. His research encompasses theory, design, and implementation of efficient and flexible signal processing systems for various purposes. He is one of the founders of the company Signal Processing Devices Sweden $\mathrm{AB}$ that sells advanced signal processing solutions. He is the author or coauthor of four books and some 170 international journal and conference papers.

Dr. Johansson is the coauthor of three papers that have received best paper awards and he has authored one invited paper in IEEE Transactions and four invited chapters. He has served as an Associate Editor for IEEE TRANSACTION ON CIRCUITS AND SYSTEMS I and II, IEEE TRANSACTION ON SIGNAL PROCESSING, and IEEE Signal Processing Letters. He is currently an Associate Editor of IEEE TRANSACTION ON CIRCUITS AND SYSTEMS I and the Area Editor of the Elsevier Digital Signal Processing journal, and a Member of the IEEE International Symposium on Circuits and Systems DSP track committee.

Oscar Gustafsson (S'98-M'03-SM' 10) received the M.Sc., Ph.D., and Docent degrees from Linköping University, Linköping, Sweden, in 1998, 2003, and 2008 , respectively.

He is currently an Associate Professor and the Head of the Electronics Systems Division, Department of Electrical Engineering, Linköping University. His research interests include design and implementation of DSP algorithms and arithmetic circuits. He has authored and coauthored more than 140 papers in international journals and conferences on these topics.

Dr. Gustafsson is a Member of the VLSI Systems and Applications and the Digital Signal Processing technical committees of the IEEE Circuits and Systems Society. Currently, he serves as an Associate Editor for the IEEE TRANSACTIONS ON CIRCUITS AND SYSTEMS-PART II: EXPRESS BRIEFS AND INTEGRATION, and the VLSI Journal. He has served and serves in various positions for conferences such as the International Symposium on Circuits and Systems, International Workshop on Power and Timing Modeling, Optimization and Simulation, PrimeAsia, Asilomar, Norchip, the European Conference on Circuit Theory and Design, and the International Conference on Electronics and Communication Systems.

Seb J. Savory (M'07-SM'11) received the M.Eng., M.A., and Ph.D. degrees in engineering from the University of Cambridge, Cambridge, U.K., in 1996, 1999, and 2001, respectively, and the M.Sc. (Maths) degree in mathematics from the Open University, Milton Keynes, U.K., in 2007.

His interest in optical communications began in 1991, when he joined Standard Telecommunications Laboratories, Harlow, U.K., prior to being sponsored through his undergraduate and postgraduate studies, after which he rejoined Nortel's Harlow Laboratories. In 2005, he joined the Optical Networks Group at University College London, holding a Leverhulme Trust Early Career Fellowship from 2005 to 2007, and was appointed a University Lecturer in 2007 and subsequently Reader in Optical Fibre Communication (OFC) in 2012. From June 2009 to June 2010, he was also a Visiting Professor at the Politecnico di Torino, Torino, Italy. His current research interests include digital signal processing, optical transmission subsystems, systems and networks.

Dr. Savory is the Editor-in-Chief of IEEE Photonics Technology Letters, an IEEE Photonics Society representative on the Steering Committee of OFC and will serve as a General Chair for OFC in 2015. He previously served as a Program Chair for OFC 2013, Signal Processing in Photonic Communications (SPPCom) 2012 and SPPCom 2013. In addition, he serves on the Editorial Board for IET Optoelectronics and the technical program committee for the European Conference on Optical Communication Design. He was also a Session Chair in 2013 IEEE Symp Design. Currently, he is serving as an Editorial Board Member for Elsevie Digital Signal Processing journal.
Amir Eghbali (S'08-M'11) was born in Urmia, Iran, in 1980. He received the of Science degree from the Iran University of Science and Tech ectrical engineering respectively.

of Electrical Engineering, Linköping University. Between November 200 VLSI design. Symposium on Circuits and Systems; 2007 International Symposium on I and Signal Processing and Analysis; 2011 European Conference on Cir 